\section{Early high plasma ST2, the decoy IL-33 receptor, in children undergoing hematopoietic cell transplantation is associated with the development of post-transplant diabetes mellitus}

Post-transplant diabetes mellitus (PTDM) occurs in $60 \%$ of adults post-allogeneic hematopoietic cell transplant (post-HCT) and has a negative impact on survival. ${ }^{1}$ Soluble Stimulation-2 (sST2), the decoy IL-33 receptor, is the most validated predictor of refractory acute graft-versus-host disease (aGvHD) and death post-HCT. ${ }^{2}$ In adults, day +14 plasma sST2 concentration was elevated in those who develop PTDM. ${ }^{3}$ There are no studies evaluating the incidence of PTDM and its relationship to sST2 in pediatric hematopoietic cell transplant (HCT) recipients. Here, we describe the incidence of PTDM in children. We also relate its diagnosis to complications post-HCT ( $\mathrm{aGvHD}$, veno-occlusive disease, and pediatric intensive care unit [PICU] admission), and day +100 postHCT and overall survival. Furthermore, we correlated day +14 plasma sST2 with the development of PTDM.

Institutional review board approval was obtained prior to the start of this study. Fifty-five HCT recipients, age $\leq$ 21 years, were prospectively accrued and plasma samples were collected at day +14 post-HCT. PTDM was defined as no pre-existing diabetes, a first fasting blood sugar $\geq 126 \mathrm{mg} / \mathrm{dL}$ or random blood sugar $\geq 200 \mathrm{mg} / \mathrm{dL}$ during the first 100 days post-HCT. ${ }^{1}$ Cumulative incidence function used the cohort median $(23 \mathrm{ng} / \mathrm{mL})$ of day $+14 \mathrm{sST} 2$ as a cut-off for risk categorization into high (above) and low (below). Unadjusted and adjusted hazard ratios (HR) using Akaike's Information Criterion (AIC) for multivariable selection were calculated. Time-dependent receiver operating characteristic (ROC) curves for day +14 sST2 were generated to evaluate the area under the curve (AUC) for risk of developing PTDM by days +30 and +100 post-HCT. Death was used as a competing risk.

Table 1. Demographics and patient characteristics stratified by the development of post-transplant diabetes mellitus.

$\begin{array}{ccc}\text { PIDM } & \text { PIDM } \\ (n=17) & P\end{array}$

Demographics

\begin{tabular}{lccc} 
Sex (female) & $5(29)$ & $13(34)$ & 0.7260 \\
\hline Age (years) & $7.7(0.8,16.0)$ & $10.0(4.9,13.8)$ & 0.3769 \\
Diagnoses & & & \\
Lymphoid malignancy & $7(41)$ & $9(24)$ & 0.2315 \\
Myeloid malignancy & $3(18)$ & $14(38)$ & 0.3930 \\
Nonmalignant & $7(41)$ & $3.0(3.0,5.0)$ & 0.2830 \\
\hline Disease Risk Index score & $3.0(2.0,5.0)$ & 25 & 10 \\
Conditioning regimen & 15 & 3 & 0.7642 \\
Full & 2 & 0 & $15(39)$ \\
Reduced & $6(35)$ & \\
Nonmyeloablative & &
\end{tabular}

Total body irradiation

$6(35)$

$15(39)$

0.7642

Donor source

\begin{tabular}{|c|c|c|c|}
\hline Bone marrow & $6(35)$ & $26(68)$ & \\
\hline Cord blood & $10(59)$ & $9(24)$ & 0.0363 \\
\hline Peripheral blood & $1(6)$ & $3(8)$ & \\
\hline
\end{tabular}

Donor status

\begin{tabular}{lccc} 
Matched related & $3(18)$ & $18(47)$ & $12(32)$ \\
Matched unrelated & $7(41)$ & $8(21)$ & 0.3219 \\
Mismatched unrelated & $7(41)$ & $0(0)$ & 0.0259 \\
$2^{\text {nd }}$ transplant & $3(18)$ & $17.7(15.8,22.4)$ & 0.8275 \\
\hline Body mass index at transplant & $19.1(16.4,21.6)$ & na & na \\
Time to PTDM (median, range) & $19.0(3.0,44.0)$ & $14.0(14.0,14.0)$ & na* \\
Time to acute GrHD (median, range) & $60.0(32.0,70.0)$ & $5(13)$ & 0.4348
\end{tabular}

PTDM or any time during the

first 100 days post HCT

Time to corticosteroids (median, range)

Values displayed are frequencies with (\%) for categorical variables and were compared using $\chi^{2}$ or Fisher's Exact test where appropriate. \% are rounded to the nearest whole number. Continuous variables are displayed in medians (interquartile ranges) and were compared with Wilcoxon rank sum test. ${ }^{*} P$-value not obtained due to only one data point for time to aGvHD for the no PTDM group. PTMD: post-transplant diabetes mellitus; aGvHD: acute graft-versus-host disease; na: not analysed. 
A

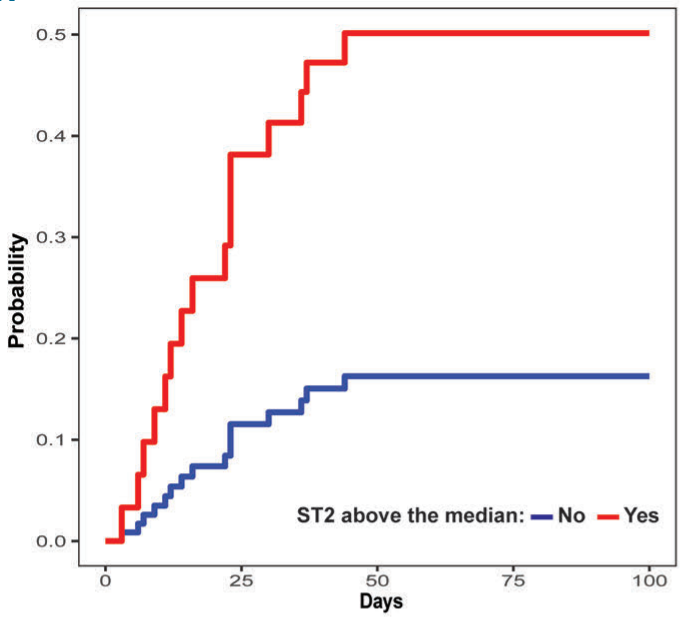

C

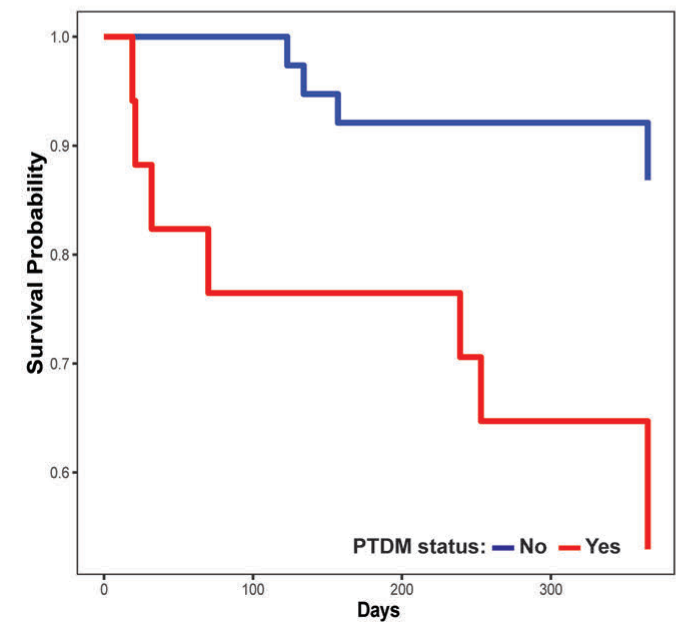

B

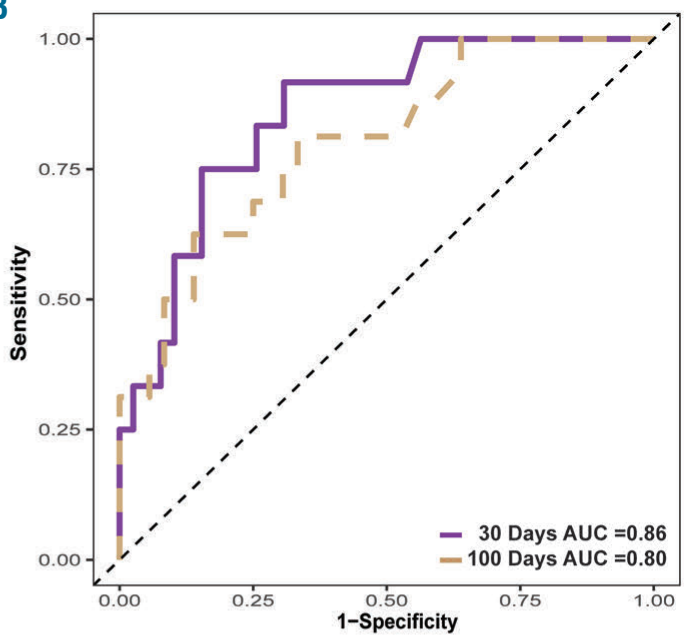

D

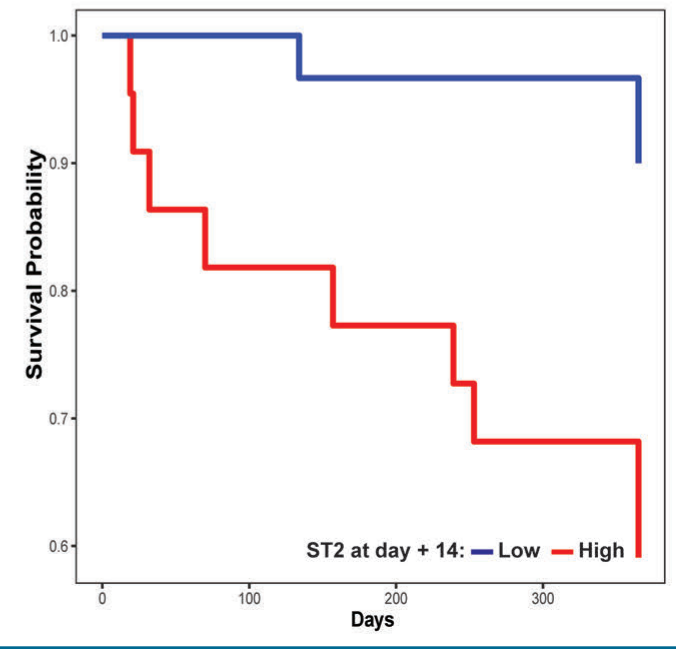

Figure 1. sST2 measured on day +14 post-allogeneic hematopoietic cell transplant is associated with the development of post-transplant diabetes mellitus and survival. (A) Cumulative incidence function graphs for risk based on sST2 level at day +14 post-allogeneic hematopoietic cell transplant (post-HCT). Cumulative incidence of the development of post-transplant diabetes mellitus (PTDM) comparing those at high risk (sST2 > cohort median of $23 \mathrm{ng} / \mathrm{mL}$ ) to those at low risk (sST2 < cohort median of $23 \mathrm{ng} / \mathrm{mL}$ ). Death was used as a competing risk. (B) Time dependent receiver operating characteristic curve for sST2 measured at day +14 post-HCT. Time dependent receiver operating characteristic curves using sST2 levels measured on day +14 post-HCT to determine the risk of developing PTDM by day 30 and 100 post-HCT. Death was used as a competing risk. (C) Survival curve by PTDM status. Survival stratified by the development of PTDM. Those in who developed PTDM ( $n=17$ ), illustrated in red, had worse 1-year survival that those who did not developed PTDM ( $n=38$ ), illustrated in blue. Log-rank $P$-value $=0.0035$. (D) Survival curve by high and low SST2. Survival stratified by high sST2 (above the cohort median of $23 \mathrm{ng} / \mathrm{mL}$ ) and low sST2 (below $23 \mathrm{ng} / \mathrm{mL}$ ). Those with a high sST2 on day +14 had a worse probability of survival at 1 year than those who had a low sST2. Log-rank $P$-value=0.0072.

PTDM was diagnosed in $31 \%$ of children. The median day to development of PTDM was day +19 post-HCT (range: 3.0, 44.0 days). Demographics including sex, age, malignant diagnosis, matched or related donor status, disease risk index, high-dose corticosteroids ( $\geq 1 \mathrm{mg} / \mathrm{kg}$ methylprednisolone equivalent for at least 72 hours), and body mass index did not differ significantly (Table 1). Those who received a cord blood transplant were more likely to develop PTDM $(P=0.0363)$. All patients $(n=3)$ who underwent a second transplant developed PTDM $(P=0.0259)$. Outcomes were worse in those who developed PTDM (Table 2). Univariate analysis showed those with PTDM had increased rates of aGvHD $(P=0.0085)$. Four patients diagnosed with PTDM also had aGvHD grade I-IV with a median time to development of 60 days (range: 32-70 days) (Table 1). Only three of these patients had a maximum grade II-IV aGvHD. Two of these three patients were diagnosed with aGvHD prior to the PTDM diagnosis (two and three days prior respectively). One patient was diagnosed with aGvHD 36 days after the diagnosis of PTDM. There was no difference in the use of high dose steroids and the median time to steroid use was not statistically different (Table 1). Those with PTDM were also more likely to be admitted to the intensive care unit $(71 \%$ vs. $8 \%, P<0.0001)$ and more likely to receive mechanical ventilation $(53 \%$ vs. $5 \%, P=0.001)$. Finally, those with PTDM also had decreased survival at 100 days $(P=0.0070)$ as compared to patients who did not develop PTDM. While not reaching statistical significance, more patients with PTDM died of non-relapsed mortality (NRM) than those who did not develop PTDM $(P=0.14)$.

Those who developed PTDM had a higher mean sST2 level at day +14 post HCT: $95.1 \pm 90.0$ compared to those who did not develop PTDM: 28.2 $\pm 28.7, P=0.0002$. Also, examining sST2 as a continuous variable, we found a higher risk for PTDM (hazard ratio $[\mathrm{HR}]=1.013$, 95\% 
Table 2. Outcomes and multivariate analyses of SST2 levels based on post-transplant diabetes mellitus status.

\begin{tabular}{|c|c|c|c|}
\hline & $\begin{array}{l}\text { PTDM } \\
(n=17)\end{array}$ & $\begin{array}{c}\text { No PTDM } \\
(n=38)\end{array}$ & $P$ \\
\hline \multicolumn{4}{|l|}{ HCT Outcomes, n (\%) } \\
\hline aGvHD grade I-IV & $5(29)$ & $1(3)$ & 0.0085 \\
\hline Veno-occlusive disease & $5(29)$ & $3(8)$ & 0.0908 \\
\hline \multicolumn{4}{|l|}{ Survival Outcomes, n (\%) } \\
\hline Survival 100 days post-HCT & $13(77)$ & $38(100)$ & 0.0070 \\
\hline Survival 1 year post-HCT & $10(59)$ & $34(90)$ & 0.0239 \\
\hline Overall survival & $9(53)$ & $32(84)$ & 0.0208 \\
\hline \multicolumn{4}{|l|}{ Causes of Death } \\
\hline Relapsed Mortality & $3(38)$ & $5(83)$ & 0.14 \\
\hline Nonrelapsed Mortality* & $5(63)$ & $1(17)$ & \\
\hline \multicolumn{4}{|c|}{ Day +14 sST2 Biomarker > 23 ng/mL } \\
\hline Unadjusted HR (95\% CI) & $3.92(1.42-10.84)$ & reference & 0.0085 \\
\hline Adjusted HR (95\% CI) & $4.06(1.55-10.62)$ & reference & 0.0043 \\
\hline
\end{tabular}

Values displayed are frequencies with (\%) for categorical variables and were compared using Chi Squared or Fisher's Exact test where appropriate. \% are rounded to the nearest whole number. HR: hazard ratio and CI: confidence interval were adjusted based on Akaike's Information Criterion (AIC) for second transplant and dono source. * Causes of non-relapsed mortality (NRM) included infection ( $\mathrm{n}=1$ ), acute graft-versus-host disease (aGVHD) ( $\mathrm{n}=2$ ), and SOS ( $=2$ ) in the PTDM group and pulmonary hemorrhage in the group with no PTDM.HCT: hematopoietic cell transplant; n: number.

confidence interval $[\mathrm{CI}]: 1.01-1.02, P<0.0001)$. We then, in an effort to identify a clinically useful cutpoint, categorized patients into high and low risk, using the median sST2 level as a cutpoint. Illustrated with cumulative incidence function graphs (Figure 1A), those with high sST2 on day +14 had a higher risk of developing PTDM ( $\mathrm{HR}=3.92$, 95\% CI: 1.42-10.84, unadjusted $P=0.0085)$. Using death as a competing risk and adjusting for donor source and second transplant, high sST2 was still strongly associated with PTDM occurrence within 100 days post-HCT $(\mathrm{HR}=4.06,95 \%$ CI: $1.55-10.62$, adjusted $P=0.0043)$. A time-dependent ROC curve, with a competing risk of death, demonstrated that sST2 had an excellent ability to discriminate the development of PTDM by 30 (AUC $=0.86$ ) and 100 days (AUC $=0.80$ ) post-HCT (Figure 1B). Survival curves, estimated by Kaplan-Meier curves method, showed that patients with PTDM had significantly lower survival than those who did not, log-rank $P$-value for the difference of 0.0035 (Figure 1C). Those with a high sST2 also had a lower survival, similar to the survival curve by PTDM status, logrank $P$-value of 0.0072 (Figure 1D). To examine if sST2 was different even earlier in the transplant course, we investigated samples at day -7 (pre-HCT) on 10 no PTDM versus 18 PTDM patients. In this small sample size, the mean +/- standard error of the mean (SEM) was $23.3 \pm 6.7$ for those with PTDM compared to $30.3 \pm 13.7$ in those who did not develop PTDM, the $P$-value $=0.7184$ was not statistically significantly different.

This is one of the first studies reporting the incidence of PTDM in children post-HCT, which unexpectedly appears common. Furthermore, to our knowledge, this is the first manuscript to report an association between sST2 and PTDM in a pediatric cohort. In adults with hyperglycemia post-HCT, studies have found increased risk for aGvHD and nonrelapsed mortality., ${ }^{4,5}$ Our study found similar results in a pediatric population. Children with PTDM have increased $\mathrm{aGvHD}$ and the need for critical care, and subsequently decreased overall survival. Surprisingly, but similar to a recent adult study, ${ }^{6}$ there was not an association of the use of high dose corticosteroids with PTDM. This suggests that HCT acutely modifies glucose homeostasis in recipients too young to have changes induced by age and other common factors. Perhaps, as suggested in non-transplant diabetes, PTDM is induced by inflammation resulting in insulin resistance. ${ }^{7}$ Supporting this is our finding that children with high plasma sST2, as early as 14 days post-HCT, had a significantly higher risk to develop PTDM. This is similar to adults with type-2 diabetes and PTDM. ${ }^{1,8}$ Importantly, in our cohort, similar to the adult cohort, the best statistical model did include high day +14 sST2 but not aGvHD. ${ }^{3}$ These data suggest that the ST2/IL-33 pathway plays a role in maintaining the metabolic equilibrium after HCT. Fasting pre-HCT glucose levels have been shown to identify PTDM susceptibility in adults ${ }^{6}$ and testing for it pre-HCT is crucial. However, fasting glucose levels are sometimes difficult to perform in children younger than 10 years and we believe that early testing of sST2 levels may help identify those at higher risk for PTDM development and help guide the frequency of blood glucose monitoring, particularly in this younger population. As our adult patients, we recommend testing for pre-HCT fasting glucose levels in children. In addition, early testing of sST2 levels may help identify those at higher risk for PTDM development in children difficult to evaluate by pre-HCT fasting glucose levels with the goal of early prevention and treatment of peripheral insulin resistance, which may improve survival.

Courtney M. Rowan, ${ }^{1}$ Alicia M. Teagarden, ${ }^{1}$ Daniel T. Cater, ${ }^{1}$ Elizabeth A.S. Moser, Giorgos Baykoyannis and Sophie Paczesny

'Department of Pediatrics, Indiana University School of Medicine and ${ }^{2}$ Department of Biostatistics, Indiana University, Indianapolis, IN, USA

Correspondence: COURTNEYROWAN - coujohns@iu.edu

SOPHIE PACZESNY - sophpacz@iu.edu

doi:10.3324/haematol.2019.222992.

Information on authorship, contributions, and financial \& other disclosures was provided by the authors and is available with the online version of this article at wWw. haematologica.org. 


\section{LETTERS TO THE EDITOR}

\section{References}

1. Griffith ML, Jagasia MH, Misfeldt AA, et al. Pretransplantation CPeptide level predicts early posttransplantation diabetes mellitus and has an impact on survival after allogeneic stem cell transplantation. Biol Blood Marrow Transplant. 2011;17(1):86-92.

2. Vander Lugt MT, Braun TM, Hanash S, et al. ST2 as a marker for risk of therapy-resistant graft-versus-host disease and death. N Engl J Med. 2013;369(6):529-539

3. Johnpulle RA, Paczesny S, Jung DK, et al. Metabolic complications precede alloreactivity and are characterized by changes in suppression of tumorigenicity 2 signaling. Biol Blood Marrow Transplant. 2017;23(3):529-532.

4. Fuji S, Kim SW, Mori S, et al. Hyperglycemia during the neutropenic period is associated with a poor outcome in patients undergoing myeloablative allogeneic hematopoietic stem cell transplantation.
Transplantation. 2007;84(7):814-820.

5. Sheean PM, Freels SA, Helton WS, Braunschweig CA. Adverse clinical consequences of hyperglycemia from total parenteral nutrition exposure during hematopoietic stem cell transplantation. Biol Blood Marrow Transplant. 2006;12(6):656-664.

6. Engelhardt BG, Savani U, Jung DK, et al. New-onset post-transplant diabetes mellitus after allogeneic hematopoietic cell transplant is initiated by insulin resistance, not immunosuppressive medications. Biol Blood Marrow Transplant. 2019;25(6):1225-1231.

7. Esser N, Legrand-Poels S, Piette J, Scheen AJ, Paquot N. Inflammation as a link between obesity, metabolic syndrome and type 2 diabetes. Diabetes Res Clin Pract. 2014;105(2):141-150.

8. Fousteris E, Melidonis A, Panoutsopoulos G, et al. Toll/interleukin-1 receptor member ST2 exhibits higher soluble levels in type 2 diabetes, especially when accompanied with left ventricular diastolic dysfunction. Cardiovasc Diabetol. 2011;10:101. 